\title{
Familial Non-Medullary Thyroid Carcinoma in Pediatric Age: Our Surgical Experience
}

\author{
Claudio Spinelli ${ }^{1}$ Irene Piccolotti ${ }^{1} \cdot$ Alessia Bertocchini $^{1} \cdot$ Riccardo Morganti $^{2} \cdot$ Gabriele Materazzi $^{3}$. \\ Massimo Tonacchera $^{4} \cdot$ Silvia Strambi ${ }^{1}$
}

\begin{abstract}
Background The purpose of the article was to evaluate the existence of significant clinical, pathological and prognostic differences between familial and sporadic form of pediatric non-medullary thyroid carcinoma, in order to tailor the therapeutic strategy to be adopted for patients with family history.

Methods We analyzed the records of 76 pediatric patients that underwent surgery for differentiated thyroid cancer from 2014 to 2019 at the Surgical Pathology Department of the University of Pisa, Italy. Among these, 20 (26,3\%) had positive family history (familial non-medullary thyroid carcinoma-FNMTC group) while 56 (73.7\%) were affected by sporadic forms (sporadic non-medullary thyroid carcinoma-SNMTC group).

Results In our study, the correlation between the FNMTC and the SNMTC group showed no difference in terms of tumor features like multifocality, bilaterality, capsular/extracapsular invasion and the presence of vascular emboli. A statistical significance, on the other hand, was revealed by observation of clinical outcomes, such as distant metastasis $(p=0,022)$, persistence of disease $(p=0,054)$ and necessity of radioiodine sessions $(p=0,005)$.

Conclusions These findings suggest that family history may have an independent role on the outcome, expressing its action through an intrinsic more aggressive biological behavior. Therefore, familial non-medullary thyroid carcinoma in children represents a nosological entity that requires an accurate pre-operative evaluation, an adequate surgical strategy and a careful follow up.
\end{abstract}

Claudio Spinelli

c.spinelli@dc.med.unipi.it

1 Pediatric and Adolescent Surgery Division, Department of Pathology Surgery, University of Pisa, Via Paradisa 2, 56124 Pisa, Italy

2 Section of Statistics, Department of Clinical and Experimental Medicine, University of Pisa, Via Paradisa 2, 56124 PisaPisa, Italy

3 Endocrine Surgery Division, Department of Surgical Pathology, University of Pisa, Via Paradisa 2, 56124 Pisa, Italy

4 Endocrinology Unit, Department of Clinical and Experimental Medicine, University of Pisa, Via Paradisa 2, 56124 Pisa, Italy

\section{Introduction}

Differentiated thyroid carcinoma accounts (DTC) for 0.5 to $3 \%$ of all childhood carcinomas and represents $90 \%$ of all thyroid cancers in childhood [1], while the medullary thyroid carcinoma (MTC) affects only $10 \%$ of pediatric cancer population [2]. Environmental risk factors for developing DTC are exposure to ionizing radiation, iodine deficiency, nodular and autoimmune disease and obesity [3-5]. With regard to genetic causes, the most frequently affected genes are RET / PTC, BRAF and RAS [6-8]. By now, pediatric thyroid carcinoma is considered a biologically different cancer from the adult one and there is a growing interest in identifying risk factors that can predict its aggressiveness and prognosis [9]. Family history is 
emerging among the risks and the negative prognostic factors, not only within the MEN form, of which the family and hereditary nature is widely known, but also in the differentiated, non-MEN, thyroid cancer [10]. This allows to divide differentiated thyroid carcinomas in two clinical entities: familial non-medullary thyroid carcinoma (FNMTC) and sporadic non-medullary thyroid carcinoma (SNMTC) [11]. According to the literature, familial cancers can account for about $10-15 \%$ of differentiated thyroid cancer of follicular derivation [12]. Hereditary forms of thyroid tumors have peculiar clinical and prognostic characteristics [13]. In fact, patients affected by FNMTC have a higher risk of bilaterality, multifocality, lymph node/remote invasion and recurrence [14], with a reduced disease-free survival [15]. Recognition of such patients would allow for more radical surgical treatment and accurate post-operative follow up [11]. Although family history has been recognized as a predictor of an unfavorable prognosis, there is still much to study on the weight of inheritance on outcome. Despite the increasing focus on this form of thyroid cancer, causative genes of FNMTC are still unknown; some studies suggest that the FNMTC has an autosomal dominant behavior with incomplete penetrance and variable expressivity $[16,17]$. Due to the poor knowledge of the genetic basis of the FNMTC, a genetic test for FNMTC is not yet available [18, 19]. This study aims to analyze the weight of genetic inheritance on the tumor's anatomo-clinical features, on the surgical management and on the outcome of pediatric patients, in order to assess the importance of inheritance as an independent prognostic factor.

\section{Materials and methods}

This is a retrospective study reviewing pediatric patients ( $\leq 18$ years old) affected by Differentiated Thyroid Carcinoma (DTC), diagnosed and treated in the Pediatric and Adolescent Surgery Unit and in the Endocrine Surgery Unit of the University of Pisa (Italy) from January 2014 to December 2019. We included in the study 76 patients with a diagnosis of DTC confirmed on definitive histological examination by the Pathological Anatomy Unit of the same University. Collected records for each patients are family history of thyroid cancer, sex, age, pre-existing benign thyroid pathology (multinodular goiter and Hashimoto thyroiditis), clinical presentation, tumor size, surgery (thyroidectomy, hemithyroidectomy, lymphadenectomy of the central and lateral compartment, unilateral or bilateral), cervical lymph nodes metastases, distant metastases, multifocality, bilaterality, capsular invasion, extrathyroid invasion, neoplastic vascular emboli, histological examination, post-operative complications (recurrent laryngeal nerve palsy, transient hypocalcaemia, permanent hypocalcaemia), persistence or recurrence of disease, number of radiotherapy sessions received. Ultrasonography was performed in all patients before surgery, to determine tumor location, size and lymph node status; if the lesion had ultrasound features suggestive for thyroid cancer, we associated a fine needle aspiration biopsy (FNAB) of the mass. When a cervical lymph node was suspected of lymph node metastasis, we performed an additional FNAB to decide whether the patient needed therapeutic neck dissection or not. Thyroid function tests, including triiodiothyronine (T3), free T3 (FT3), thyroxine (T4), free T4 (FT4), thyroglobulin (Tg), thyroid-stimulating hormone (TSH), anti-peroxidase antibody (TPOAb) and anti-thyroglobulin antibody $(\mathrm{TgAb})$, calcitonin and calcemia were performed before surgery. Other imaging studies, such as CT / RMN and radioactive iodine uptake test (RAIU test), were performed to evaluate lung metastases and mediastinal lymph nodes. The post-operative follow up was performed every 3 months for the first 2 years with clinical tests, ultrasound and blood tests (calcemia, TPOAb, TgAb, T3, FT3, T4, FT4, TSH and Tg levels) and then every 6 months. A chest $\mathrm{x}$-ray or CT/RMN scan was obtained once a year. Patients were considered "disease-free" at follow up if they had a suppressed serum $\mathrm{Tg}<1 \mathrm{ng} / \mathrm{mL}$, no detectable $\operatorname{TgAb}$, and no structural evidence of disease. Patients with $\mathrm{Tg}$ values $\geq 1 \mathrm{ng} / \mathrm{mL}$, or stimulated Tg values $\geq 2 \mathrm{ng} / \mathrm{mL}$, or any evidence of disease on imaging (ultrasonography or computed tomography scan, radioactive iodine scan) or biopsy-proven disease (cytology or histology) within one year from initial treatment were considered as having persistent disease. A recurrence was defined as new biochemical, structural, or functional evidence of disease that was detected after at least one year [20, 21].

Patients were divided into two groups based on the presence or absence of family history for thyroid cancer, emerging from the anamnestic data: Familial Non-Medullary Thyroid Carcinoma (FNMTC) and Sporadic NonMedullary Thyroid Carcinoma (SNMTC). We included in the study all subjects with diagnosis of FNMTC ( $\leq 18$ years old) with at least one first (parents) or second (siblings or uncles) degree relative diagnosed with differentiated thyroid carcinoma [22, 23].

\section{Data analysis}

Categorical data were summarized by absolute and relative frequency, quantitative ones by mean or median and standard deviation or range. To compare the variable "central compartment" or "lateral compartment" with the qualitative risk factors, chi-square test and z-test for two 
Table 1 Comparison of the clinical factors between the FNMTC group and the SNMTC group

\begin{tabular}{lll}
\hline & FNMTC $(20)$ & SNMTC (56) \\
\hline Familiarity & & \\
Maternal line & $13(65 \%)$ & \\
Paternal line & $6(30 \%)$ & \\
Both (Maternal-Paternal line) & $1(5 \%)$ & $16(28.6 \%)$ \\
Sex & & $40(71.4 \%)$ \\
Male & $6(30 \%)$ & $14.99( \pm 1.6)$ \\
Female & $14(70 \%)$ & \\
Age & & $9(16.1 \%)$ \\
Average & $15.45( \pm 1.8)$ & $47(83.9 \%)$ \\
Previous benign thyroid disease & & \\
Yes & $5(25 \%)$ & $35(62.5 \%)$ \\
No & $15(75 \%)$ & $21(37.5 \%)$ \\
Clinical presentation & & \\
Thyroid nodule & $15(75 \%)$ & $32(57.1 \%)$ \\
Thyroid nodule + limphadenopathy & $5(25 \%)$ & $24(42.9 \%)$ \\
Tumor size & & 0.286 \\
$<2$ cm & $12(60 \%)$ & 0.484 \\
$>2$ cm & $8(40 \%)$ & 0.967 \\
\hline
\end{tabular}

proportions were used, while for the quantitative risk factors Student's t test for independent samples (two tailed) was applied. Significance was set at 0.05. All analyzes were performed with SPSS version 26 technology (Chicago, Illinois, USA).

\section{Results}

The average age of onset of DTC in our 76 patients was 15.14 years (range $8-18$ ). The average age in the FNMTC group was 15.45 years (range 10-18), while in the SNMTC group was 14.99 years (range $8-18)(p=0,286)$. The patients consisted of 22 males $(29 \%)$ and 54 females (71\%). Among the FNMTC subjects there were 14 female $(70 \%)$ and 6 males $(30 \%)$; in the SNMTC sample, females were $40(71.4 \%)$ and males $16(28.6 \%)(\mathrm{p}=0,868)$. Of the 76 patients examined, 20 patients $(26.3 \%)$ belonged to the FNMTC group. Of these patients, 5 (6.6\% of the total) had first-degree inheritance for thyroid cancer, while $15(19.7 \%$ of the total) had second-degree inheritance. Of the 20 patients with hereditary thyroid cancer, 13 (65\%) were familial along the maternal line, $6(30 \%)$ from the paternal line, while one $(5 \%)$ patient had a positive family history of carcinoma on both inherit lines. This comparison was statistically significant, with $\mathrm{p}<0,001$. Pre-existing benign thyroid pathology was found in $5(25 \%)$ patients in the FNMTC group, while in $9(16 \%)$ in the SNMTC one $(p=0,584)$. No significant differences were found between the two groups as regards the parameters: clinical presentation, tumor size, surgery, lymph nodes metastases, multifocality, bilaterality, capsule invasion, extrathyroidal invasion, neoplastic embolism and recurrences. Regarding histological examination, classical variant of papillary carcinoma was present in 15 cases $(75 \%)$ of FNMTC and in 36 cases $(64.3 \%)$ of SNMTC $(p=0,550)$ while follicular variant in 2 cases $(10 \%)$ of FNMTC and in 10 cases $(17.85 \%)$ of the SNMTC one; the analysis of histological subtypes did not reveal a significant difference between the two groups (Tables 1, 2). Regarding outcomes, interesting statistically significant differences emerged for distant metastasis (all located at the lung level), persistence of disease and number of radioiodine (RAI) sessions. Distant metastases were found in $3(15 \%)$ familial subjects, while were absent in the patients affected by sporadic carcinoma $(p=0,022)$. Persistence of disease occurred in $6(30 \%)$ patients of the FNMTC group and in $4(7.1 \%)$ of SNMTC group $(p=0,054)$. The difference between the average number of RAI sessions for patient in the two groups is statistically significant, with $p=0,005$ (Table 3). 
Table 2 Comparison of the surgical and anatomo-pathological factors between the FNMTC group and the SNMTC group. Statistics: frequency $(\%)$ or mean $( \pm \mathrm{sd})$

\begin{tabular}{|c|c|c|c|}
\hline Factor & FNMTC (20) & SNMTC (56) & $\mathrm{p}$ value \\
\hline Surgery & & & 0.758 \\
\hline Lobectomy & $1(5 \%)$ & $6(10.7 \%)$ & \\
\hline Thyroidectomy (Tx) & $19(95 \%)$ & $50(89.3 \%)$ & \\
\hline Lymphadenectomy (CC or LC) & & & 0.392 \\
\hline No lymphadenectomy & $10(50 \%)$ & $36(64.3 \%)$ & 0.392 \\
\hline Yes lymphadenectomy (L) & $10(50 \%)$ & $20(35.7 \%)$ & \\
\hline $\mathrm{L}-\mathrm{Tx}+\mathrm{cc}$ unilateral & $6(30 \%)$ & $4(7.1 \%)$ & 0.264 \\
\hline $\mathrm{L}-\mathrm{Tx}+\mathrm{lc}$ unilateral & $0(0 \%)$ & $1(1.8 \%)$ & 0.175 \\
\hline $\mathrm{L}-\mathrm{Tx}+\mathrm{cc}+\mathrm{lc}$ unilateral & $3(15 \%)$ & $14(25 \%)$ & 0.875 \\
\hline $\mathrm{L}-\mathrm{Tx}+\mathrm{cc}+\mathrm{lc}$ bilateral & $1(5 \%)$ & $1(1.8 \%)$ & 0.506 \\
\hline Metastatic Cervical Compartments $(N+)$ & & & 0.654 \\
\hline No lymphonodal metastases & $4(20 \%)$ & $7(12.5 \%)$ & \\
\hline Yes Lymphonodal metastases (LM) & $16(80 \%)$ & $49(87.5 \%)$ & \\
\hline LM-Central compartment (CC) & $8(40 \%)$ & $19(33.9 \%)$ & 0.887 \\
\hline LM-Lateral compartment (LC) & $4(20 \%)$ & $15(26.8 \%)$ & 0.832 \\
\hline LM-Both central and lateral compartments $(\mathrm{CC}+\mathrm{LC})$ & $3(15 \%)$ & $14(25 \%)$ & 0.627 \\
\hline LM-CC + LC bilateraly & $1(5 \%)$ & $1(1.8 \%)$ & 0.838 \\
\hline \multicolumn{4}{|l|}{ Lymph nodes removed } \\
\hline Average lymph nodes removed $(\mathrm{N}) /$ patient & $12.2( \pm 2.8)$ & $15.13( \pm 3.7)$ & 0.002 \\
\hline Average metastatic lymph nodes $(\mathrm{N}+) /$ patient & $4( \pm 2.6)$ & $5.05( \pm 2.7)$ & $\mathbf{0 . 0 3 8}$ \\
\hline Removed/metastatic lymph nodes rate & $0.327( \pm 0.1)$ & $0.333( \pm 0.1)$ & 0.820 \\
\hline Multifocality & & & 0.967 \\
\hline Yes & $8(40 \%)$ & $24(42.9 \%)$ & \\
\hline No & $12(60 \%)$ & $32(57.1 \%)$ & \\
\hline Bilaterality & & & 0.901 \\
\hline Yes & $3(15 \%)$ & $11(19.6 \%)$ & \\
\hline No & $17(85 \%)$ & $45(80.4 \%)$ & \\
\hline Capsular invasion & & & 0.650 \\
\hline Yes & $17(85 \%)$ & $43(76.8 \%)$ & \\
\hline No & $3(15 \%)$ & $13(23.2 \%)$ & \\
\hline Extrathyroid invasion & & & 0.181 \\
\hline Yes & $7(35 \%)$ & $20(35.7 \%)$ & \\
\hline No & $13(65 \%)$ & $36(64.3 \%)$ & \\
\hline Neoplastic vascular emboli & & & 0.519 \\
\hline None & $8(40 \%)$ & $29(51.8 \%)$ & \\
\hline Yes (Y) & $12(60 \%)$ & $27(48.2 \%)$ & \\
\hline $\mathrm{Y}>4$ & $5(25 \%)$ & $17(30.3 \%)$ & 0.375 \\
\hline $\mathrm{Y}<4$ & $7(35 \%)$ & $10(17.9 \%)$ & - \\
\hline \multicolumn{4}{|l|}{ Histology } \\
\hline Follicular Carcinoma & $0(0 \%)$ & $0(0 \%)$ & $>0.05$ \\
\hline Papillar Carcinoma (PC) & $20(100 \%)$ & $56(100 \%)$ & - \\
\hline PC-Classical Variant & $15(75 \%)$ & $36(64.3 \%)$ & 0.550 \\
\hline PC-Follicular variant & $2(10 \%)$ & $10(17.9 \%)$ & 0.642 \\
\hline PC-Solid & $1(5 \%)$ & $5(8.8 \%)$ & 0.939 \\
\hline PC-High cell & $1(5 \%)$ & $3(5.4 \%)$ & 0.602 \\
\hline PC-Diffuse sclerosing variant & $1(5 \%)$ & $2(3.6 \%)$ & 0.699 \\
\hline
\end{tabular}


Table 3 Comparison of the post-operative complications and prognostic factors between the FNMTC group and the SNMTC group. Statistics: frequency $(\%)$ or mean $( \pm \mathrm{sd})$

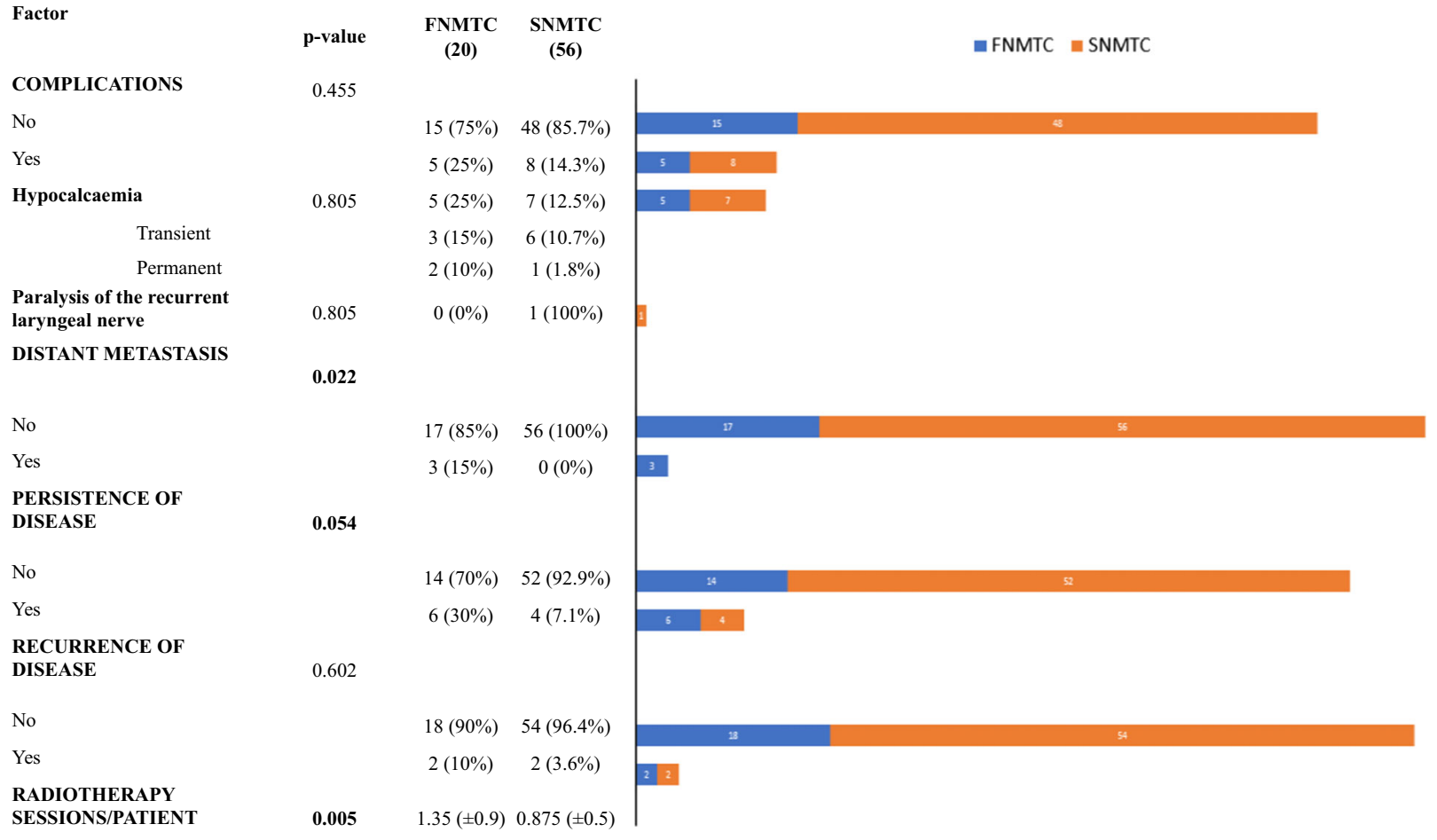

\section{Discussion and conclusions}

The present study compares a group of pediatric subjects with Familial Non-Medullary Thyroid Carcinoma with a control group consisting of pediatric subjects affected by a sporadic form.

Regarding the clinicopathological features, in our casuistry, there were no significant differences between the two groups. We registered a prevalence of pre-existing benign thyroid pathology in familial forms of $25 \%$, which is lower than data reported in the literature [18, 24]; for example, Haggie Mazeh et al. [13] described a prevalence of pre-existing benign thyroid disease of $45 \%$.

According to the literature [25-27], the clinical presentation of the tumor as well as its size at diagnosis was equally distributed in the two groups $(p=0,967)$. Regarding surgical treatment, no major use of thyroidectomy was found in subjects with familial disease (95\%) compared to sporadic forms $(89 \%)(p=0.758)$. Lymphadenectomy was performed on half of the familial subjects and on $35 \%$ of the sporadic cases. Regarding lymph node metastases, there were no significant differences in the two groups, and this was also confirmed by the multivariate statistical analysis. Similar results were found for the number of lymph nodes removed, the number of metastatic lymph nodes and their relationship. In our study, there was no difference in the analysis of multifocality, bilaterality, capsular and extracapsular invasion and presence of vascular emboli, and these results are in conflict with other studies we found in the literature $[14,15]$ that reported a more aggressive local presentation for familial carcinomas. Uchino et al. [14] stated that, compared to the patients with sporadic disease, the FNMTC patients were more likely to have multifocal disease ( $40.7 \%$ for FNMTC vs. $28.5 \%$ for SNMTC, $p<0.0001$ ), while in our study, the prevalence of multifocality was similar in the two groups (40\% for FNMTC vs. $42.9 \%$ for SNMTC, $p=0.967$ ).

Interesting data, on the other hand, emerged in our study from the analysis of clinical outcomes, such as complications, persistence and recurrence of disease, necessary radioiodine sessions and distant metastasis. In the literature, there is a lack of studies on post-operative complications of thyroidectomy in the pediatric population and in particular of specific studies on the family form of differentiated thyroid carcinoma. In our study, FNMTC group had a slightly higher post-operative complication rate but this difference was not statistically significant $(p=0.455)$. Incidence of distance metastases in FNMTC group in our 
casuistry was $15 \%$ higher compared to control group, with a statistically significant difference $(p$ value $=0,022)$. Robenshtok et al. [28] reported a higher incidence (8\%) in patients with a familial form than in those with a sporadic form $(5 \%)$, while other authors [29, 30] have found no increase in distant metastasis in familial form.

In our study, the incidence of persistence of disease in patients with FNMTC was significantly higher than in patients affected by SNMTC ( $p=0,054)$ and this finding is in line with recent literature [13, 29, 31-33]. Other interesting data concern the average number of necessary radiotherapy sessions in the two groups, with a statistically significant difference $(p$ value $=0.005)$. From another point of view, as highlighted by Hillenbrand A. et al. [34], a greater number of radiotherapy sessions for FNMTC confirms a reduction in disease-free survival in these subjects, in accordance with the increase in distant metastases and persistence of disease. The discrepancy between these data and those that emerged from the analysis of local invasiveness features suggests that inheritance is an independent negative prognostic factor with respect to the local invasiveness of the tumor, which was comparable to the sporadic forms and this is probably due to a different genetic biology of the tumor [35]. Although the local extent of the tumor has not been enhanced by inheritance, the forms of FNMTC presented a worse outcome in terms of risk of distant metastasis, persistence of disease and average number of radiotherapy sessions for patient. These findings suggest that family history may have an independent role on the outcome, expressing its action through an intrinsic more aggressive biological behavior. Therefore, familial non-medullary thyroid carcinoma in children represents a nosological entity that requires an accurate pre-operative evaluation, an adequate surgical strategy and a careful follow-up.

The study is limited by its retrospective and registrybased nature, and thus some data can be missing. In addition, since the familiarity was found from the anamnesis collected by the patient's parents, often without any personal medical documentation, some distortions due to recall mistakes in the family history can have been included. Nevertheless, we hope that our study can pave the way for other research on the familial form of differentiated thyroid carcinoma in pediatric age.

Open Access This article is licensed under a Creative Commons Attribution 4.0 International License, which permits use, sharing, adaptation, distribution and reproduction in any medium or format, as long as you give appropriate credit to the original author(s) and the source, provide a link to the Creative Commons licence, and indicate if changes were made. The images or other third party material in this article are included in the article's Creative Commons licence, unless indicated otherwise in a credit line to the material. If material is not included in the article's Creative Commons licence and your intended use is not permitted by statutory regulation or exceeds the permitted use, you will need to obtain permission directly from the copyright holder. To view a copy of this licence, visit http://creativecommons. org/licenses/by/4.0/.

Funding Open access funding provided by Università di Pisa within the CRUI-CARE Agreement.

\section{References}

1. Hung W, Sarlis NJ (2002) Current controversies in the management of pediatric patients with well-differentiated nonmedullary thyroid cancer: a review. Thyroid 12(8):683-702

2. Balachandar S, La Quaglia M, Tuttle RM et al (2016) Pediatric Differentiated Thyroid Carcinoma of Follicular Cell Origin: Prognostic Significance of Histologic Subtypes. Thyroid 26(2):219-226

3. Faggiano A, Coulot J, Bellon N et al (2004) Age-dependent variation of follicular size and expression of iodine transporters in human thyroid tissue. J Nucl Med 45(2):232-237

4. Shibata Y, Yamashita S, Masyakin VB et al (2001) 15 years after Chernobyl: new evidence of thyroid cancer. Lancet 358(9297): 1965-1966

5. Ron E, Lubin JH, Shore RE et al (1995) Thyroid-cancer after exposure to external radiation-a pooled analysis of 7 studies. Radiat Res 141(3):259-277

6. Davies L, Morris LG, Haymart M et al (2015) American association of clinical endocrinologists and american college of endocrinology disease state clinical review: the increasing incidence of thyroid cancer. Endocr Pract 21(6):686-696

7. Jung CK, Little MP, Lubin JH et al (2014) The increase in thyroid cancer incidence during the last four decades is accompanied by a high frequency of BRAF mutations and a sharp increase in RAS mutations. J Clin Endocrinol Metabolism 99(2):E276-E285

8. Fagin JA (2004) Challenging dogma in thyroid cancer molecular genetics-role of RET/PTC and BRAF in tumor initiation. J Clin Endocrinol Metabolism 89(9):4264-4266

9. Spinelli C, Strambi S, Rossi L et al (2016) Surgical management of papillary thyroid carcinoma in childhood and adolescence: an Italian multicenter study on 250 patients. J Endocrinol Invest 39(9):1055-1059

10. Spinelli C, Di Giacomo M, Costanzo S et al (2010) Role of RET codonic mutations in the surgical management of medullary thyroid carcinoma in pediatric age multiple endocrine neoplasm type 2 syndromes. J Ped Surg 45(8):1610-1616

11. Sippel RS, Caron NR, Clark OH (2007) An evidence-based approach to familial nonmedullary thyroid cancer: screening, clinical management, and follow-up. World J Surg 31(5):924-933

12. Nose V (2008) Familial non-medullary thyroid carcinoma: an update. Endocr pathol 19(4):226-240

13. Mazeh H, Sippel RS (2013) Familial nonmedullary thyroid carcinoma. Thyroid 23(9):1049-1056

14. Uchino S, Noguchi S, Kawamoto H et al (2002) Familial nonmedullary thyroid carcinoma characterized by multifocality and a high recurrence rate in a large study population. World J Surg 26(8):897-902

15. Musholt TJ, Musholt PB, Petrich T et al (2000) Familial papillary thyroid carcinoma: genetics, criteria for diagnosis, clinical features, and surgical treatment. World J Surg 24(11):1409-1417

16. Moses W, Weng J, Kebebew E (2011) Prevalence, clinicopathologic features, and somatic genetic mutation profile in familial versus sporadic nonmedullary thyroid cancer. Thyroid 21(4):367-371 
17. Xiong Y, Zhang L, Holloway AK et al (2011) MiR-886-3p regulates cell proliferation and migration, and is dysregulated in familial non-medullary thyroid cancer. PLoS ONE 6(10):e24717

18. Bevan S, Pal T, Greenberg CR et al (2001) A comprehensive analysis of MNG1, TCO1, fPTC, PTEN, TSHR, and TRKA in familial nonmedullary thyroid cancer: confirmation of linkage to TCO1. J Clin Endocrinol Metab 86(8):3701-3704

19. Cavaco BM, Batista PF, Martins C et al (2008) Familial nonmedullary thyroid carcinoma (FNMTC): analysis of fPTC/PRN, NMTC1, MNG1 and TCO susceptibility loci and identification of somatic BRAF and RAS mutations. Endocr Relat Cancer 15(1):207-215

20. Sapuppo G, Tavarelli M, Belfiore A et al (2019) Time to separate persistent from recurrent differentiated thyroid cancer: different conditions with different outcomes. J Clin Endocrinol Metabolism 104(2):258-265

21. Bates MF, Lamas MR, Randle RW et al (2018) Back so soon? Is early recurrence of papillary thyroid cancer really just persistent disease? Surgery 163(1):118-122

22. Fallah M, Pukkala E, Tryggvadottir L et al (2013) Risk of thyroid cancer in first-degree relatives of patients with non-medullary thyroid cancer by histology type and age at diagnosis: a joint study from five Nordic countries. J Med Genet 50(6):373-382

23. Pal T, Vogl FD, Chappuis PO et al (2001) Increased risk for nonmedullary thyroid cancer in the first degree relatives of prevalent cases of nonmedullary thyroid cancer: a hospital-based study. J Clin Endocrinol Metabolism 86(11):5307-5312

24. Loh KC (1997) Familial nonmedullary thyroid carcinoma: a meta-review of case series. Thyroid 7(1):107-113

25. Davies L, Welch HG (2014) Current thyroid cancer trends in the United States. JAMA Otolaryngol Head Neck Surg 140(4):317-322

26. Davies L, Welch HG (2006) Increasing incidence of thyroid cancer in the United States, 1973-2002. JAMA 295(18):2164-2167
27. Ho AS, Davies L, Nixon IJ et al (2015) Increasing diagnosis of subclinical thyroid cancers leads to spurious improvements in survival rates. Cancer 121(11):1793-1799

28. Robenshtok E, Tzvetov G, Grozinsky-Glasberg S et al (2011) Clinical characteristics and outcome of familial nonmedullary thyroid cancer: a retrospective controlled study. Thyroid 21(1):43-48

29. Alsanea O (2000) Familial nonmedullary thyroid cancer. Curr Treat Options Oncol 1(4):345-351

30. Park YJ, Ahn HY, Choi HS et al (2012) The long-term outcomes of the second generation of familial nonmedullary thyroid carcinoma are more aggressive than sporadic cases. Thyroid 22(4):356-362

31. Mazeh H, Benavidez J, Poehls JL et al (2012) In patients with thyroid cancer of follicular cell origin, a family history of nonmedullary thyroid cancer in one first-degree relative is associated with more aggressive disease. Thyroid 22(1):3-8

32. Spinelli C, Rallo L, Morganti R et al (2019) Surgical management of follicular thyroid carcinoma in children and adolescents: a study of 30 cases. J Pediatric Surg 54(3):521-526

33. Spinelli C, Tognetti F, Strambi S et al (2018) Cervical lymph node metastases of papillary thyroid carcinoma, in the central and lateral compartments, in children and adolescents: predictive factors. World J Surg 42(8):2444-2453

34. Hillenbrand A, Varhaug JE, Brauckhoff M et al (2010) Familial nonmedullary thyroid carcinoma-clinical relevance and prognosis: a European multicenter study ESES Vienna presentation. Langenbecks Arch Surg 395(7):851-8

35. Capezzone M, Marchisotta S, Cantara S et al (2008) Familial non-medullary thyroid carcinoma displays the features of clinical anticipation suggestive of a distinct biological entity. Endocr Relat Cancer 15(4):1075-1081

Publisher's Note Springer Nature remains neutral with regard to jurisdictional claims in published maps and institutional affiliations. 